\title{
ANÁLISE DA CICLICIDADE IMPRESSA NO REGISTRO SEDIMENTAR DA SEÇÃO NEO-APTIANA / EOCENOMANIANA (FM. RIACHUELO) NA BACIA DE SERGIPE-ALAGOAS.
}

\author{
JOSÉ MILTON CRONEMBERGER MENDES
}

\begin{abstract}
CYCLICITY ANALYSIS OF THE NEO-APTIAN / EOCENOMANIAN SECTION (RIACHUELO FM.) - SERGIPE/ ALAGOAS BASIN. Qualitative analysis using well logs and inter basin correlation confirms the importance of orbital mechanisms in determining cyclic patterns in the Neo-Aptian/Eocenomanian stratigraphic succession in the Sergipe/Alagoas Basin. The use of statistics softwares applied to the data resulted in a high degree of similarity between both depositional and orbital cycles in the "Milankovitch band".

Quantitative cyclicity analysis in the studied section allowed a detailed stratigraphic subdivision, not possible using traditional tools like seismic and biostratigraphic data. The estimate of sedimentary rates and depositional/erosional hiatuses during the deposition of the mapped sedimentary sequences, using the identified cycles and biostratigraphic data, made possible a better understanding of the studied stratigraphic section.
\end{abstract}

Resumo Análise qualitativa através de perfis de poços e correlação com seções equivalentes entre bacias confirma a importância das variações orbitais na determinação dos padrões cíclicos encontrados na sucessão estratigráfica Neo-Aptiana / Eocenomaniana da Bacia de Sergipe-Alagoas. A utilização de softwares estatísticos aplicados aos dados resultou num alto grau de similaridade entre os ciclos deposicionais e os ciclos orbitais na "faixa de Milankovitch".

A análise quantitativa da ciclicidade na seção estudada permitiu um detalhamento estratigráfico que não seria possível através de ferramentas tradicionais como sísmica e bioestratigrafia. A estimativa de variações de taxas de sedimentação e de hiatos deposicionais/erosivos durante a deposição das sequiências mapeadas, utilizando-se os ciclos identificados e as datações bioestratigráficas disponíveis, permitiu um melhor entendimento da evolução estratigráfica da seção analisada.

INTRODUÇÃO A análise de ciclicidade para esse intervalo estratigráfico na porção sergipana da bacia de Sergipe-Alagoas é uma tentativa preliminar de se estabelecer conexão entre a aparente ciclicidade observada em perfis elétricos/radioativos/acústicos e possível causa orbital como mecanismo responsável por variações climáticas, que imprimiram no registro estratigráfico essas feições. $O$ objetivo principal desse trabalho é tentar quantificar a duração dos diversos ciclos observados tantos através de perfis como em afloramentos, tirando conclusões sobre taxas de sedimentações, hiatos deposicionais e possíveis causas para variações nesses números.

A análise estratigráfica através dos conceitos de ciclicidade

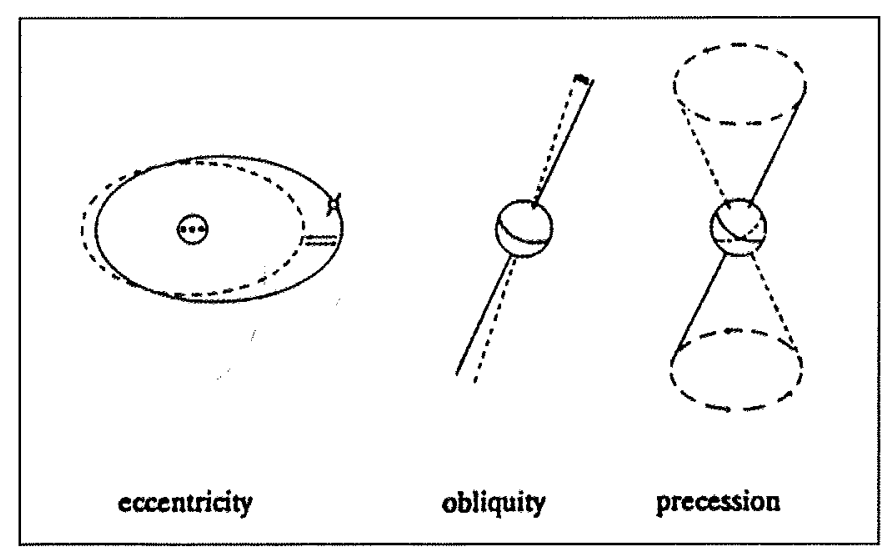

Figura 1: Variações orbitais: os ciclos regidos pela excentricidade possuem como valores principais 100.000 anos e 400.000 anos, aqueles associados à obliqüidade possuem período de 41.000 anos e os ciclos precessionais possuem periodos principais de 19.000 anos e 23.000 anos (Fisher et al, 1991) é um fascinante campo onde a Estratigrafia está adquirindo notáveis avanços nos últimos anos, se constituindo em poderosa ferramenta de correlação e de detalhamento estratigráfico.

Apesar de ter tido especial avanço após os conceitos da estratigrafia de seqüências, a análise da ciclicidade no registro sedimentar já vem sendo objeto de considerações desde o início do século XIX. Adhemar (1842) já associava variações climáticas a causas orbitais; Croll (1875) correlacionou duas importantes épocas de glaciações com 100 mil anos e 210 mil anos à excentricidade da órbita da Terra; Gilbert,em 1900, associou a interação entre variações orbitais e processos geológicos como responsáveis pela ritmicidade do registro estratigráfico; Milankovitch, em 1941, desenvolveu as idéias de Croll, fundamentando-as (apud Fischer \& Bottjer, 1991). Seus cálculos para as periodicidades devidas a causas orbitais ficaram no intervalo de 104-105 anos. Segundo Arthur \& Garrison (1986), os cálculos de 400 mil anos e 100 mil anos para a duração de ciclos regidos pela excentricidade, 41 mil anos para ciclos de obliquidade e de 19 mil anos a 23 mil anos para ciclos de precessão (todos na "faixa de Milankovitch"), foram realizados por Berger et al (1992) que os considerou como relativamente estáveis para os últimos 5 milhões de anos (fig.1).

Coogan (1967), analisando ciclicidade em seqüências carbonáticas, já realçava a importância da mesma na previsão de ocorrência/recorrência de "unidades dos ciclos".

Desde 1985, aumentou consideravelmente o número de trabalhos que se dedicam à análise da ciclicidade (estratigrafia de eventos de alta resolução/bioeventos, causas orbitais $X$ seqüências sedimentares, etc), utilizando-se os mais variados fenômenos: físicos (erupções vulcânicas, fluxos de massa, subsidência tectônica, etc), oceanográficos (correntes, variações de salinidade, eventos anóxicos, etc), químicos (análises de isótopos estáveis, teores de carbonatos, etc), climáticos (variações climáticas associadas a causas orbitais, tempestades, etc), biológicos (produtividade orgânica, mortandade em massa, etc), 
etc. Segundo Seilacher (1991), atualmente, mesmo os eventos mais imprevisíveis, tais como impactos de grandes meteoritos, são reinvidicados a ocorrer com uma certa periodicidade.

Ainda é muito questionada a utilização da ciclicidade na análise de intervalos estratigráficos. As principais críticas referem-se a problemas no próprio empilhamento estratigráfico, onde seria mais comum a ausência do que a presença do registro sedimentar. Outras referem-se a limitações na determinação de idades absolutas ("quanto mais antigas, mais imprecisas"), a alterações diagenéticas, a problemas relacionados com os cálculos das periodicidades das variações orbitais (imprecisão para intervalos com mais de 5 milhões de anos de idade), etc.

Independentemente de problemas, que obviamente podem e devem ocorrer, a utilização criteriosa dos conceitos e a escolha apropriada da seção a ser analisada tornam essa técnica uma poderosa ferramenta de detalhamento estratigráfico.

Mesmo em intervalos sem datações bioestratigráficas ou sem fósseis-índices, essa técnica permite um detalhamento cronoestratigráfico dezenas de vezes maior do que as técnicas bioestratigráficas tradicionais, tornando-se de fundamental importância para refinamentos estratigráficos, inclusive ao nível de reservatório.

Berger et al. (1992) apresentam cálculos para as variações de tempo que ocorrem nos diversos períodos relacionados à "faixa de Milankovitch", ao longo do tempo geológico. Para a faixa de idade da seção em estudo, as variações são desprezíveis (da ordem de $2 \%$ ), considerando-se as incertezas com que trabathamos tanto em termos de datações relativas como absolutas.

\section{Metodologia}

Dados Paleontológicos A seção analisada possui um bom nível de detalhamento bioestratigráfico. Utilizou-se como padrão o zoneamento através de foraminíferos realizado por Koutsoukos (1989), onde o intervalo objeto desta dissertação encontra-se

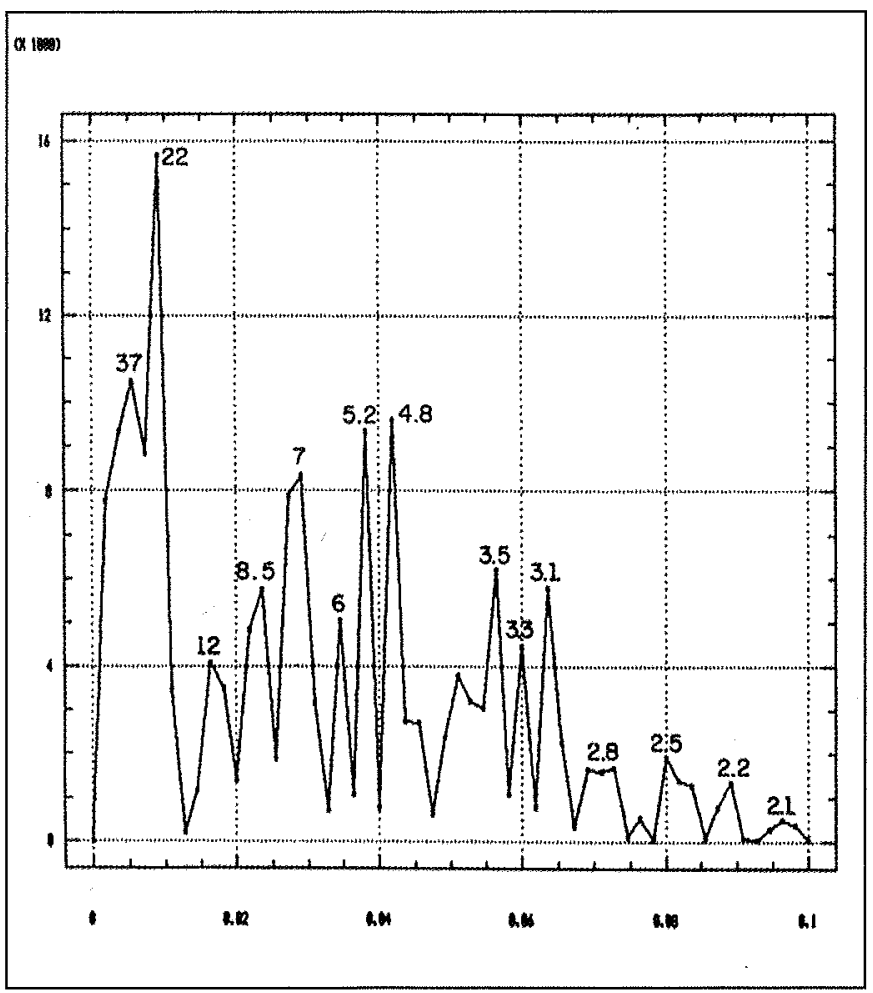

Figura 2: Periodograma correspondendo à Seq. I do poço 4. Os valores plotados nas freqüencias mais significativas representam espessuras em metros. subdividido em nove biozonas: desde a "Ap-1" (Aptiano mais tardio) até a "Ce-1" (Cenomaniano mais inicial), com cada biozona compreendendo entre 0,5 a 4,0 milhões de anos. Pelas correlações de Koutsoukos (op cit.), esse intervalo compreende aproximadamente 15,0 milhões de anos (entre 95 e 110 milhões de anos atrás).

Apesar de existirem, na área do presente estudo, apenas seis poços datados em tal nível de detalhe, onde a seção analisada encontra-se "completa" (em termos de biozonas) ou apenas com o intervalo aptiano preservado, e, além disso, esses poços encontrarem-se, em sua maioria, concentrados na parte sul da área, é relativamente segura a extrapolação desses dados para os demais poços da área, devido às informações da sísmica, de perfis dipmeter e, principalmente, através de correlações das curvas de raios gama (GR), resistividade (RILD), sônico (DLT), etc.

Quantificação da Ciclicidade As três seqüências definidas serão analisadas individualmente. A Seqüência I, compreende as biozonas Ap-2, Ap-1, e Al-1 (Aptiano mais tardio/Albiano mais inicial), totalizando aproximadamente 4 milhões de anos; a Seqüência II engloba as biozonas Al-2, Al-3 e parte da Al-4 (Eo/meso Albiano), com aproximadamente 7 milhões de anos; a Seqüência III compreende as biozonas Ce-1, Al-6, Al-5 e parte da Al-4 (Neo-Albiano/Cenomaniano mais inicial), com aproximadamente 4 milhões de anos.

O tratamento estatístico usado na determinação quantitativa

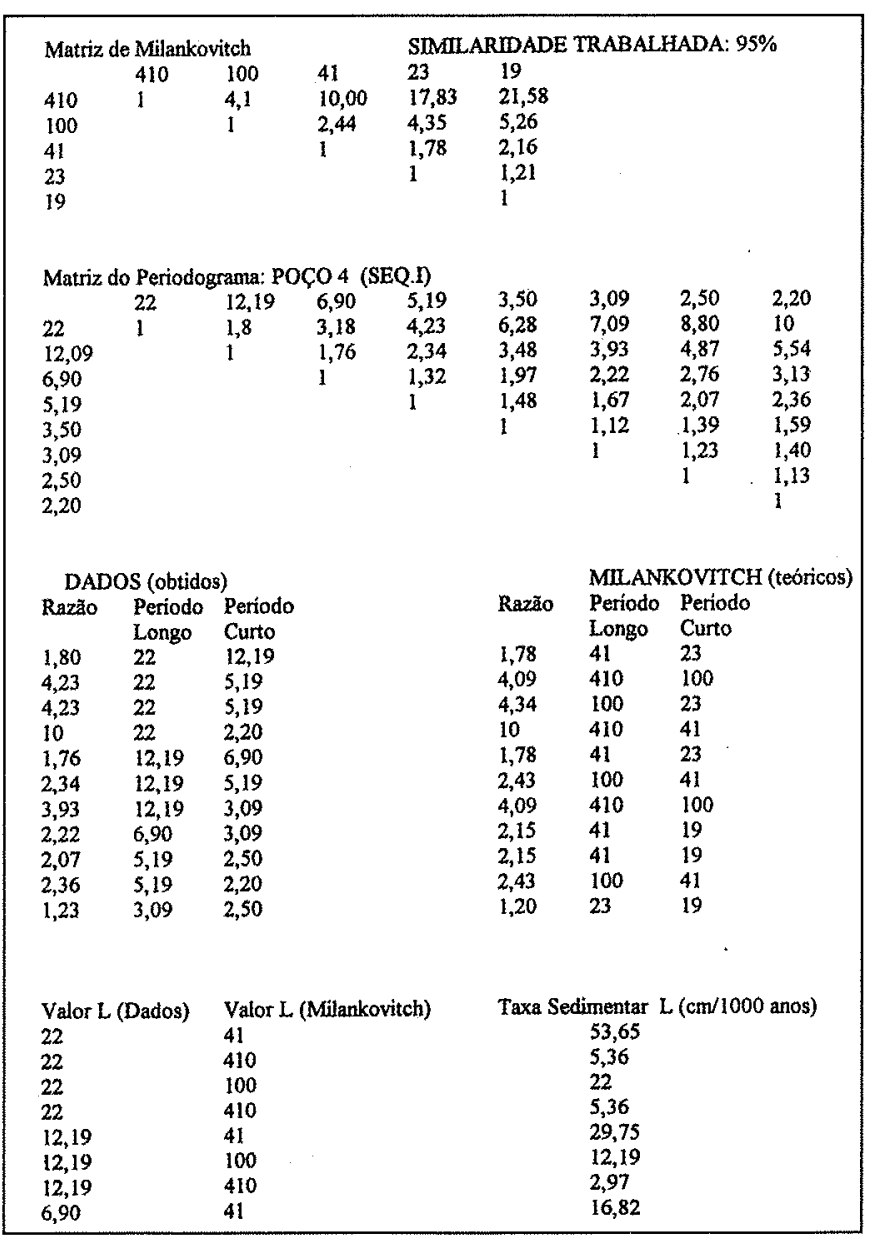

Figura 3: A correlação dos diversos períodos obtidos com causas orbitais pode ser realizada através do programa "Matrizes". Para cada associação realizada o programa calcula taxas de sedimentação, em centímetros por mil anos. 
dos diversos ciclos presentes na seção analisada foi obtido com a utilização do programa STATGRAPHICS. Simplificadamente, o programa produz periodogramas (Fig.2) onde são plotados os valores de amplitudes das senóides constatadas nas ordenadas, e as freqüências das oscilações nas abscissas (cycles/sampling interval). O comprimento de onda ou período de cada freqüência "significativa" - aquelas com altos valores/picos nas ordenadas, é obtido dividindo-se o intervalo de amostragem (nesse caso de $0.2 \mathrm{~m}$ ) pelo número de ciclos obtido nas abscissas. Esses valores são obtidos com maior precisão em tabelas listadas pelo programa. Maiores detalhes sobre os procedimentos e as bases teóricas desse tipo de análise poderão ser obtidos em Azambuja Filho (1990, p.68/76).

Esse tipo de tratamento pode ser realizado com qualquer parâmetro que esteja variando de maneira cíclica, independentemente se em um arranjo simétrico ou assimétrico, ou se na escala de "microciclos" ou "megaciclos" (Duff et al.,1967). Desse modo, a variação em profundidade de teores de carbonato, valores de resistividade, raios gama, tempo de trânsito, etc, podem ser submetidos a semelhantes análises.

A correlação dos diversos períodos obtidos através dos periodogramas com as diversas causas orbitais, pode ser realizada tentativamente, ou com a utilização do programa MATRIZES que faz uma comparação entre a matriz dos dados obtidos nos periodogramas (até oito valores) e a matriz teórica da "Faixa de Milankovitch" (com cinco valores). Em geral trabalha-se com

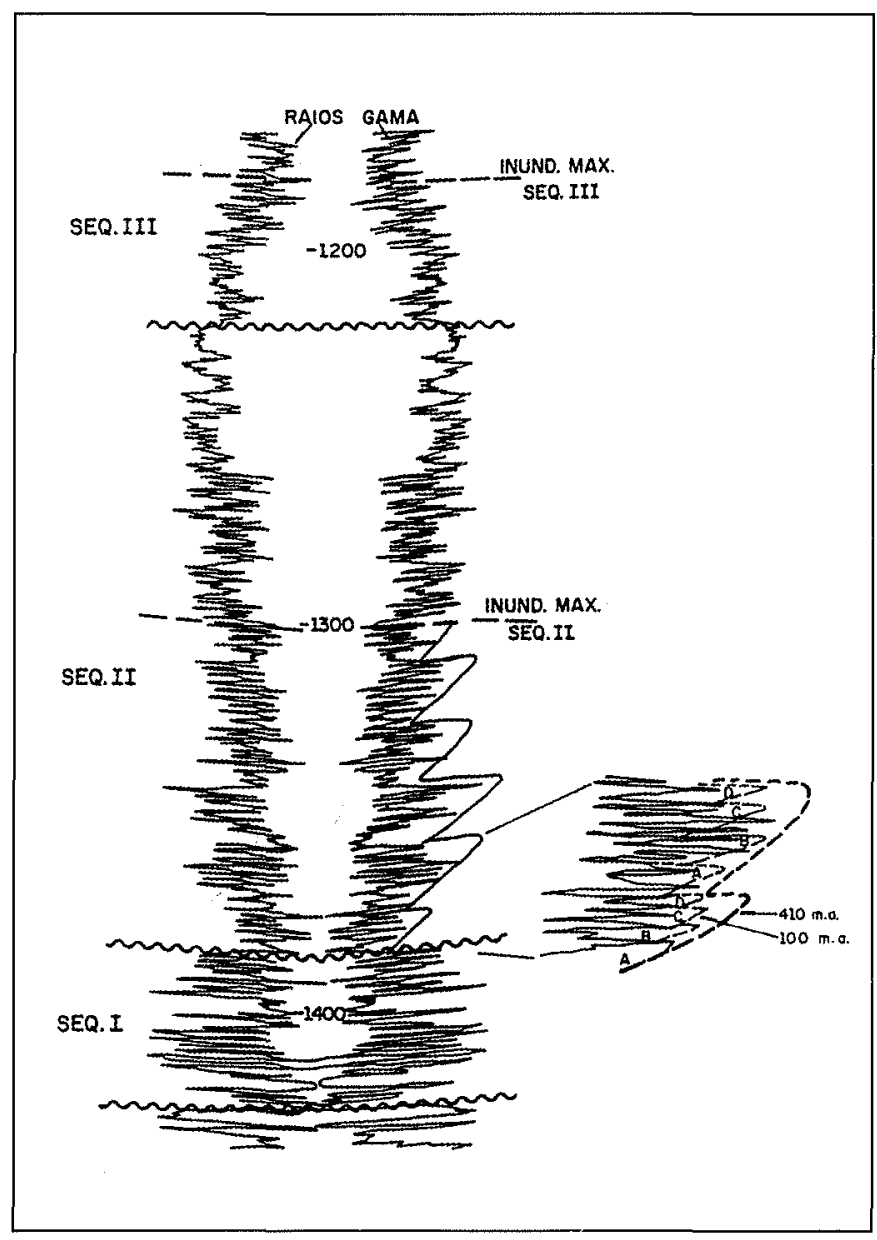

Figura 4: Padrão de empilhamento cíclico pelo $R G$ do poço 6. Intervalos com maior aproximação entre as curvas indicam melhores condições de concentração e de preservação de matéria orgânica. similaridades entre ambas de 95 a 98\%. Além dessa comparação, o programa calcula, para cada valor obtido e associado a uma causa orbital (valor teórico da. Matriz de Milankovitch), a sua equivalente taxa sedimentar média (em $\mathrm{cm} / 1000$ anos) Fig.3. Nesse caso, descartam-se os valores anomalamente altos ou baixos, escolhendo-se aqueles compatíveis com as taxas médias obtidas pela divisão da espessura da seqüência pelo seu respectivo tempo envolvido ná sua deposição.

Ciclicidade na Seção Analisada O Albiano tem se revelado como uma das principais seções onde há melhor preservação do registro cíclico, devido a causas orbitais, em todo o mundo (Fischer, inf. verbal). Em Sergipe, essa seção não foge à regra, em especial nas áreas mais distantes da borda da bacia ou em baixos regionais.

A simples visualização do padrão de variação dos valores de raios gama, resistividade, etc, nos poços situados em posições da bacia mais favoráveis à impressão da ciclicidade no registro sedimentar (Fig.4), mostra que essa seção é adequada para esse tipo de análise.

Teixeira (1969) descreveu a seção como um ciclo sedimentar transgressivo-regressivo, propondo mapas paleogeográficos para cada fase.

Koutsoukos et al. (1991) e Koutsoukos et al. (1993) sugeriram uma influência orbital/climática nos ciclos de folhelhos e calcilutitos e nas variações de espessura dos calcilutitos do intervalo mais inferior dessa seção, associando-os a variações de produtividade dos organismos calcários de águas superficiais.

A análise dos periodogramas para cada sequiência nos quatro poços estudados mostra fortes evidências da ciclicidade na faixa de Milankovitch, ficando sugerida a impressão no registro estratigráfico desde os ciclos precessionais (19 a 23 mil anos) até os ciclos de excentricidade longa (410 mil anos).

Para se ter uma idéia do potencial desse tipo de análise, basta considerarmos que a determinação/definição dos ciclos de 20 mil e 100 mil anos (precessão e excentricidade) fornece um detalhamento de 25 a 200 vezes e de 5 a 40 vezes maior que o método bioestratigráfico atual (biozonas com 0,5 a 4 milhões de anos). Wilson (1975) já enfatizava a importância da análise dos ciclos para um refinamento do método bioestratigráfico, ressaltando que eles podem definir intervalos de tempo da ordem de 20 vezes menor que os definidos pelas biozonas atuais.

A análise quantitativa da ciclicidade para os poços $3,4,5$,

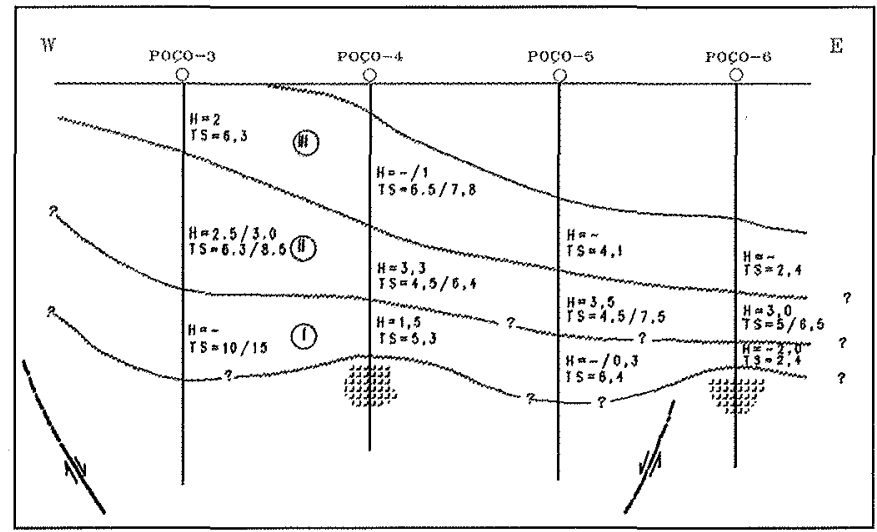

Figura 5: Evolução das taxas de sedimentação (TS em $\mathrm{cm} / 1000$ anos) ao longo do empilhamento das seqüencias $I$, II e III. TS calculadas pela análise dos ciclos em valores não descompactados. Hiatos $(H)$ em milhões de anos calculados pela contagem de ciclos e seus valores são cumulativos ao longo do empilhamento de cada seqüência. 
e 6 (Fig.5), localizados a sul de Aracaju, dá boas informações sobre a dinâmica do empilhamento estratigráfico para essa área da bacia.

A avaliação dos poços foi realizada para cada seqüência individualmente, permitindo, dessa forma, uma observação das mudanças laterais e verticais em termos de taxas de sedimentação, ocorrência ou nã́o de hiatos, etc, ao longo do tempo.

A análise da seqüência I nesses poços fornece alguns subsídios, que permitem fazer algumas inferências em termos de fisiografia da área, tectonismo, etc:

- houve um grande controle tectônico na deposição dessa seqüência: as seções aparentemente completas e com taxas de sedimentação relativamente altas nos poços 3 e 5 ,devem corresponder a maiores acomodações devido a esse fator. No poço 6, ocorre uma situação oposta: sugere-se um significativo hiato em torno de 2 milhões de anos e a taxa de sedimentação média reduz-se a menos da metade dos valores estimados para os poços 3 e 5 . Em termos de taxa de sedimentação, acredita-se que tal valor baixo seja devido, além da condensação normal da seção devido à sua posição mais distal, mas também ao controle estrutural - que reduziu dramaticamente a acomodação nessa região. Basculamentos contra a borda da bacia e movimentação halocinética podem explicar os hiatos observados nesse poço e no de número 4.

Antes de prosseguirmos com as análises das seqüências II e III, é importante que se façam as seguintes observações: a) as taxas de sedimentação calculadas são obtidas com valores de espessuras de rochas compactadas. Conseqüentemente, os valores "reais" deverão ser significativamente maiores. A não descompactação, no entanto, não está influindo nos cálculos dos hiatos, visto estes terem sido estimados com base na contagem do número de ciclos e no respectivo tempo envolvido. b) os valores estimados para os hiatos erosivos/não deposicionais não devem estar concentrados nos topos das seqüências maiores. Acredita-se que uma boa parte dos mesmos esteja nos limites das seqüências de $3 a$ ordem, mas o mais provável é que ocorram vários hiatos ao longo do empilhamento de sequiências de maior freqüência.

A seqüência II, pelo que se observa nos valores relativamente uniformes obtidos para taxas de sedimentação e hiatos, foi depositada em condições tectonicamente mais estáveis. Coincidentemente, nessa área, a presença dos carbonatos de águas rasas ocorre de maneira significativa a partir dessa unidade (mas não nesses poços usados para os cálculos de ciclicidade). Imagina-se que a fisiografia da bacia nesse período era menos acidentada do que na época da deposição da sequiência I, apesar de continuar havendo a sua compartimentação em blocos altos (p.ex. as áreas de deposição dos carbonatos de alta energia) e baixos (p.ex. as áreas de deposição das fácies lagunares).

$\mathrm{Na}$ área do poço 3 , parece que há uma subsidência significativamente mais elevada do que nas demais, o que é refletido na taxa de sedimentação maior e em uma maior preservação da seção.

A análise da seqüência III sugere o progressivo assoreamento da topografia bacial, observando-se claramente a ocorrência de basculamento para SE e/ou soerguimento na região do poço 3 .

De modo geral, pelo que se observa da fig. 5 , a área do poço 6 foi sítio de taxas sedimentares relativamente menores durante a deposição das sequiências I e III, como era de se esperar por sua localização mais distal à borda e por uma provável diminuição de acomodação devido a estar em um alto estrutural e à halocinese, mas durante a deposição da seqüência II observa-se um deslocamento de depocentro em direção à área desse poço, registrando-se significativo aumento da taxa de deposição.

$\mathrm{Na}$ área do poço 4, as taxas sedimentares foram mais baixas do que nos poços vizinhos 3 e 5 ao longo do período de deposição das seqüuencias I e II, invertendo-se a tendência durante a deposição da seqüência III. Estruturação devido à tectônica envolvendo embasamento e à halocinese deve ser a responsável por esta inversão.

A diminuição de acomodação na área do poço 3, durante o período de deposição da sequiência III, já deve ser reflexo do processo de basculamento da bacia para sudeste, provocado por subsidência térmica. A continuação desse processo de basculamento foi o responsável pela erosão/não deposição dessa sequiência em grande parte da área em estudo

De maneira geral, as taxas sedimentares encontradas para essa seção estão compatíveis com as calculadas por Garrison \& Fischer (1969, apud Hallam, 1981) para mares epicontinentais triássicos na região dos Alpes, que foram da ordem de $10 \mathrm{~cm} / 1000$ anos

Ciclicidade em Seções de Carbonatos de Plataforma Coogan (1967) observou que o fenômeno da ciclicidade era comum na natureza, questionando se a mesma era um fato do ambiente natural ou se era somente resultado da interpretação do homem. Nesse trabalho, ele define um ciclo carbonático ideal como uma cunha limitada por inconformidades ou desconformidades que comumente se estenderiam até a borda da plataforma. Na sua base ocorreria delgado depósito transgressivo que representaria uma inundação da plataforma. O topo estaria representado pelos depósitos finais da progradação. Como conseqüência, ressalta, sedimentos de fácies semelhantes separados geograficamente em uma direção "dip", seriam diácronos. Apesar de associar a ciclicidade às oscilações eustáticas, o autor não entrou em discussão sobre possíveis mecanismos responsáveis por esse processo.

Wilson (1975) classificou os ciclos de plataforma em três tipos de "padrões cíclicos": 1) ciclos de shoaling upward, essencialmente regressivos, 2) ciclos de associações de clásticos terrígenos $\mathrm{X}$ carbonatos, desde simples pares a associações mais complexas, e 3) ciclos que possuem uma fase carbonática inferior, transgressiva, e uma fase terrígena superior, regressiva. Esse autor cita quatro mecanismos principais como responsáveis pela ciclicidade:

1) subsidência constante com atuação de fatores extra bacia: variações eustáticas devido a períodos glaciais ou causadas por movimentos ao nível de tectônica de placas;

2) subsidência constante $X$ nível do mar constante, mais mecanismo extra bacia que causaria repetidos períodos de assoreamento da mesma, como por exemplo, mudanças climáticas influenciando produtividade orgânica e influxo de terrígenos, tectonismo em áreas distantes atuando nas áreas fontes da bacia, etc;

3) subsidência episódica, e

4) subsidência associada a movimentos ascendentes e descendentes da plataforma, com sedimentação contínua.

Wilson (op cit.) também ressaltou que a ciclicidade nas plataformas carbonáticas é vista como sendo causada principalmente pela eustasia, desde que a ciclicidade pode ser observada em áreas com atividades tectônicas extremamente variáveis.

Goodwin \& Anderson (1986) propuseram os "ciclos agradacionais pontuados", PAC's, que consistiriam de ciclos de 1 a 5 metros de espessura, com tendência de raseamento ascendente, e marcados por abruptas mudanças para fácies mais profundas, causadas por rápidas subidas do "nível de base", seja por eustasia, tectonismo, etc.

A análise de como os rítmos astronômicos na "faixa de Milankovitch" influenciam a deposição de carbonatos de plataforma, foi bem fundamentada por Hardie \& Shinn (1986) que subdividiram em três modos principais essa influência:

a) causando variação nos volumes de gelo continentais: a retração e a expansão desses volumes de gelo se constituiriam no mecanismo básico para as oscilações eustáticas. Para o Fanerozóico, apenas durante o Cambriano as regiões polares teriam 
ficado completamente sem continentes;

b) causando mudanças de temperaturas nos oceanos: citando Donovan \& Jones, 1979 , reportam que variações de $1^{\circ} \mathrm{C}$ provocariam oscilações eustáticas da ordem de $1 \mathrm{~m}$. Acreditam, no entanto, que tal mecanismo não influenciaria na "faixa de Milankovitch", devido à inércia das águas de fundo dos oceanos, e

c) causando mudanças climáticas nos trópicos: baixas taxas de suprimento sedimentar (produtividade orgânica) em períodos frios provocariam transgressões, e altas taxas nos períodos quentes provocariam progradação na plataforma.

Goldhammer et al. (1987) estudando carbonatos de plataforma na região das Dolomitas, nordeste da Itália, também identificaram ciclicidade na "faixa de Milankovitch", relatando que os ciclos formam um padrão análogo ao registro do nível do mar para o Pleistoceno: ciclos assimétricos maiores superimpostos por 5 ciclos menores.

Uma das constatações que se faz ao se pesquisar diferentes intervalos, com idades e ambientes tectônicos diferentes, é que a ciclicidade independe desses fatores, pois esses ciclos métricos de raseamento ascendente em depósitos carbonáticos de plataformas são encontrados desde o pré-Cambriano ao Recente, nos mais diversos tipos de regimes tectônicos (Carozzi, 1955, Coogan, 1967, Ginsburg, 1975, Wilson, 1975, Goodwin \& Anderson, 1986, Hardie \& Shinn, 1986, Goldhammer et al, 1987, entre outros). Os mecanismos responsáveis pela ciclicidade é que são, ainda, motivo de discussões: eustasia, tectonismo (local e regional/placas), clima, influxo sedimentar (tanto terrígeno como carbonático), são considerados, isoladamente ou em associação, seus principais causadores. Goldhammer et al. (op cit.) concluíram que a origem era certamente devido a oscilações eustáticas, e que a tectônica pode ter exercido importante papel - embora não seja fácil identificá-lo.

Como exemplos brasileiros de ciclicidade em plataformas carbonáticas, temos: Ojeda \& Bisol (1971) consideraram a deposição da Fm. Riachuelo como o registro de um ciclo transgressivo (membros Angico-Taquari) / regressivo (membros Maruim, Aguilhada e Angico); Della Fávera (1975) descreveu sequiências cíclicas na Fm. Preguiças da Bacia de Barreirinhas, onde a alternância de carbonatos-terrígenos foi associada a um controle climático/tectônico; Ponte \& Asmus (1976) observaram que a seção marinha da margem leste brasileira registra períodos transgressivos/regressivos, citando a seção do Eoalbiano como um dos exemplos principais; Schaller et al. (1980) consideraram que os sedimentos das formações Riachuelo e Cotinguiba, da Bacia de Sergipe-Alagoas, foram depositados de maneira cíclica, "sendo necessário levar em consideração a distribuição no tempo, caracterizar suas microfácies e observar suas relações com os terrígenos associados para se estabelecer os modelos deposicionais desses ciclos".

Spadini (1992) estudando os ciclos de deposição de carbonatos de plataforma de idade albiana da Bacia de Campos, observou que a distribuição desses resultou da atuação de três fatores: a) condicionantes regionais, que moldaram a configuração da plataforma rasa, b) oscilações do nível do mar, o que implica numa escala regional para a ciclicidade; e c) variações no ambiente deposicional, considerado como atuando em menor escala. Os ciclos de shoaling up típicos teriam na base, packstones peloidais com foraminíferos planctônicos (registro do afogamento), mostrando espessamentos de camadas para o topo, passando para packstones/grainstones oncolíticos e finalmente culminando com depósitos oolíticos, representando a deposição mais rasa.

Tagliari (1993) considerou que o controle da mistura dos siliciclásticos e carbonatos de plataforma albiana da Bacia do Espírito Santo provavelmente é conseqüência dos ciclos de Milankovitch.

Ciclicidade na Seção da Pedreira Carapeba A sucessão de ciclos da Pedreira Carapeba (Fig.6), datada como Neo-albiano (Koutsoukos,1989), tem servido de escola para várias gerações de geólogos.

A primeira impressão, é de vários ciclos de raseamento ascendente empilhados, com os níveis argilosos e margosos representando os eventos de máximas inundações. Ao se analisar com mais detalhe, no entanto, através de informações de campo e de laboratório, observa-se que esses níveis argilosos/margosos provavelmente estão representando os finais dos ciclos regressivos, pois são nessas fácies que se observam mais indicações de presença continental: madeiras carbonizadas, siliciclásticos (quartzo,

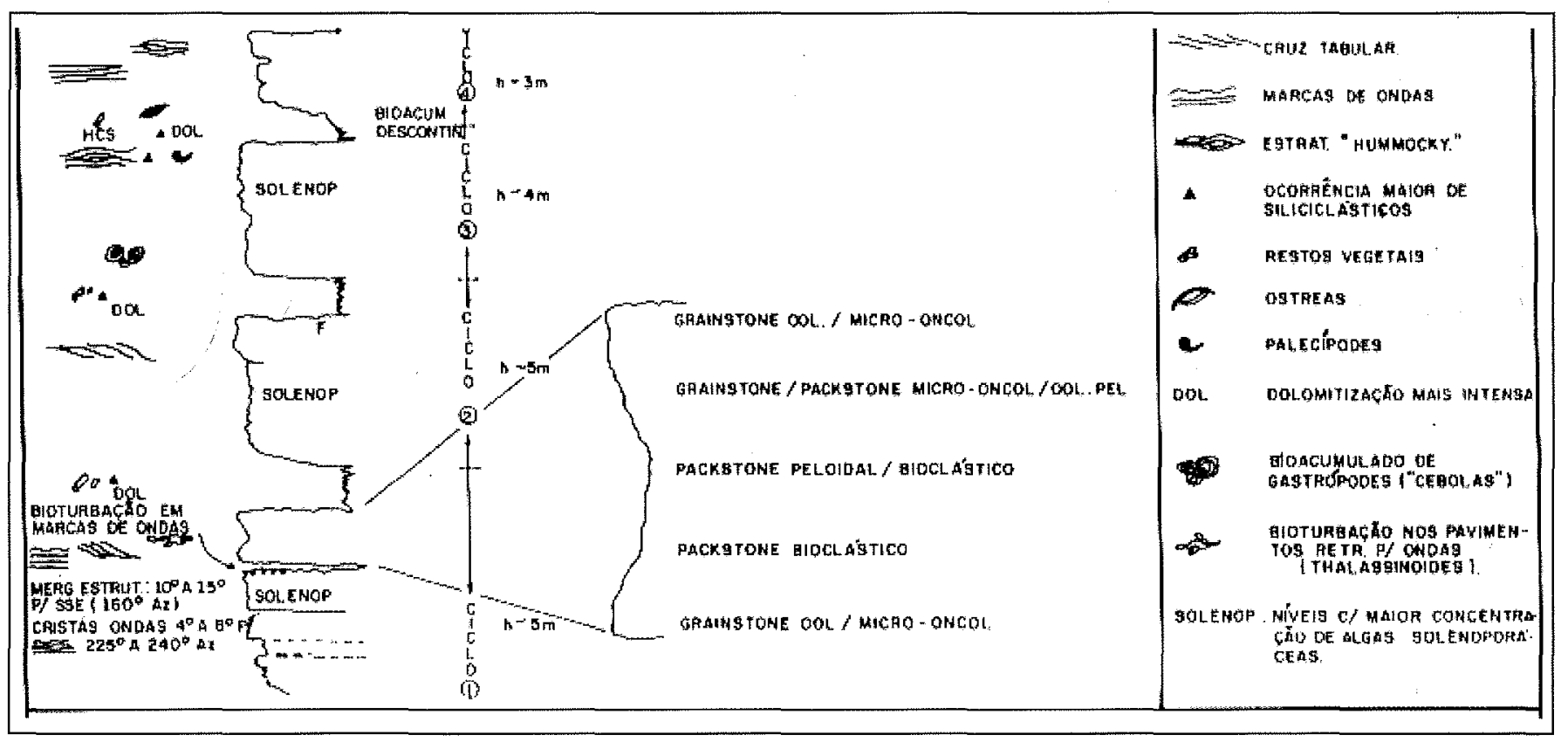

Figura 6: Perfil de "RG" simulado para o empilhamento da seção Maruim aflorante na Pedreira Carapeba. Os ciclos de raseamento ascendente de $3 m$ a $5 m$ são da magnitude dos "PAC's" de Goodwin \& Anderson (1986). 
feldspato) e intensa dolomitização, representando a chegada de água doce no sistema marinho. Os eventos transgressivos estariam representados pelos carbonatos imediatamente acima das margas, em geral packstones/grainstones peloidais ou bioacumulados (gastrópodes, pelecípodes).

Os ciclos presentes nessa pedreira apresentam espessuras da ordem de 3 a 5 metros, dentro dos limites definidos por Goodwin \& Anderson (1986) para os seus "PAC's".

$\mathrm{Na}$ análise dos possíveis mecanismos responsáveis por essa ciclicidade, depois de realizados estudos quantitativos através da utilização do programa STATGRAPHICS (ver item 2.2), concluise que a interação dos fatores clima e eustasia parece ser a responsável maior pela impressão da ciclicidade nessa seção da Bacia de Sergipe. Ficou marcante a presença dos ciclos orbitais na faixa de Milankovitch para os intervalos analisados.

Apesar das análises quantitativas não terem contemplado fácies carbonáticas de bancos rasos, os ciclos analisados com esta faixa de espessura foram associados a ciclos relacionados à obliqüidade do eixo da Terra (períodos de 41000 anos), porém em áreas com taxas de sedimentação provavelmente menores. Dessa maneira, se esses ciclos forem registro da obliqüidade, teríamos taxas de sedimentação entre 7 e $12 \mathrm{~cm} / 1000$ anos. Caso forem associados à precessão dos equinócios (19 a 23 mil anos), essas taxas subiriam para 15 a $25 \mathrm{~cm} / 1000$ anos, valores perfeitamente normais para esse tipo de sedimentação.

É inegável, no entanto, a importância do tectonismo, seja através de basculamento térmico, por atividade de falhas, uplifts, etc, na geração (ou redução) de espaço de acomodação ao longo de todo o empilhamento da seção Riachuelo, e na conformação da fisiografia da bacia, criando condições para o desenvolvimento de buildups carbonáticos lineares/orientados (Purdy et al.,1975,
Wilson, 1975). Não está no escopo deste trabalho, no entanto, a quantificação desse fator individualmente. Outro fator a ser investigado seria a fuga de sal. Pelo que se observou nas diversas seções geológicas, nas análises quantitativas de taxas de sedimentação, etc, e pelo que já observaram Estrella \& Palagi (1969), Ojeda \& Fugita (1976), Feijó (1980), entre outros, esse mecanismo tem atividade pouco significativa, atuando de modo localizado, pelo menos no que se refere à área emersa da bacia.

Um mecanismo que pode gerar "ciclos" é a própria dinâmica da plataforma rasa. Pelas características desse ambiente, pode-se ter condições bastante diferentes em termos de energia; dessa maneira, a migração lateral desses "sub-ambientes" pode provocar variações verticais de fácies que sugeririam "ciclos", apesar desse processo ocorrer de modo quase aleatório (Fig.6). Assim, tendências ascendentes de aumento ou diminuição na quantidade de micrita presente em carbonatos não devem ser associadas sempre a variações eustáticas.

Uma visualização de como poderia estar ocorrendo esse empilhamento pode ser feita pelo modelo proposto no bloco diagrama da fig.7. A região dessa pedreira configura uma área em que, periodicamente, haviam condições de deposição de calcarenitos (grainstones/packstones) em ambiente de inframaré sujeito à ação de ondas normais (e de tempestades), com freqüentes ocorrências de intervalos/níveis com intensa bioturbação refletindo "paradas" no processo sedimentar, e de margas/ calcilutitos muito argilosos. Os calcarenitos são representantes de um ambiente de praia - principalmente shoreface, mas também foreshore, e as margas de ambientes de laguna/plataforma interna. Ciclos semelhantes foram descritos por Burchette \& Wright (1992).

Em posições mais distais à borda (a SE do "ambiente Cara-

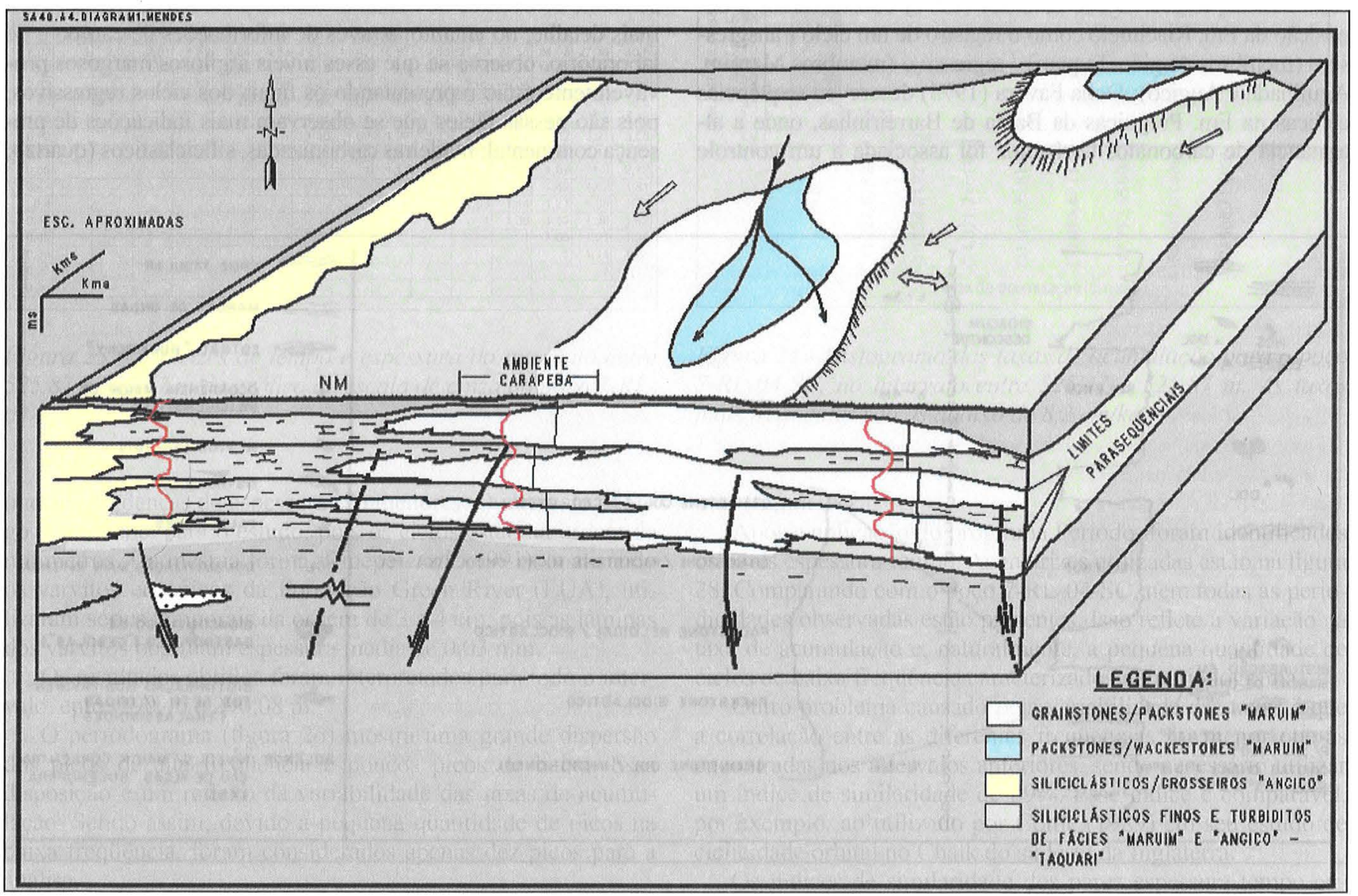

Figura 7: Diferentes padrões de empilhamento de "ciclos de raseamento ascendente", identificando-se a área da pedreira Carapeba. 
peba"), é provável que se tenha configurações diferentes para os ciclos analisados anteriomente. Assim, é normal que em algumas áreas essa ciclicidade possa ser mais semelhante ao que Spadini (1992) descreveu para ciclos de raseamento ascendente na Bacia de Campos, e em outras, mais para dentro da bacia, os eventos transgressivos sejam representados por margas, folhelhos e calcilutitos da frente dos bancos rasos (fig.7).

Agradecimentos Esse trabalho é parte de minha Dissertação de Mestrado apresentada em 1993 na UFRGS. Agradeço aos meus Orientadores, Prof. Dr. Jorge Carlos Della Fávera e Dr. Adali Spadini, pelo apoio inestimável durante o período que estive em Porto Alegre; ao Dr. Nilo Chagas de Azambuja Filho, que me incentivou e deu apoio para a realização desse estudo sobre ciclicidade na seção analisada; à Petrobras, que tornou possível esse Projeto através de apoio financeiro, de banco de dados e logístico; e à UFRGS e aos Colegas de Curso pelo excelente ambiente acadêmico e de amizade.

\section{Referências}

Arthur, M.A. \& Garrison, R.e. 1986. Cyclicity in the Milankovitch band through geologic time: an introduction. Paleoceanography, 1 (4): 369-372.

Azambuja Filho, N.C. 1990. The Oligo-Miocene turbidites and associated facies of the Campos Basin, offshore Brazil.Ph.D. thesis, University of London. 456p.

Berger, A., Loutre, M.f. \& Laskar, J., 1992. Stability of the astronomical frequencies over the Earth's history for paleoclimate studies. Science, 255, p.560-566.

Burchette, T.P. \& Wright, V.p. 1992. Carbonate ramp depositional systems. Sedimentary Geology, 79: 3-57.

Carozzi, A., 1955. Some remarks on cyclic calcareous sedimentation as an index of climatic variations. Journal of Sedimentary Petrology, 25 (1), p. 78-79.

Coogan, A.H. 1967. Recent and ancient carbonate cyclic sequences. West Texas Geol. Society, 5-16.

Della Fávera, J.C., 1975. Modelo deposicional para as formações Carbonáticas Bomfim e Preguiças - Bacia de Barreirinhas. Relat. Interno Petrobrás, $15 \mathrm{p}$.

Della Fávera, J.C., 1984. Eventos de sedimentação episódica nas bacias brasileiras. Uma contribuição para atestar o caráter pontuado do registro sedimentar. Anais do XXXIII Congr. Bras. de Geol., Rio de Janeiro, p 489-501.

Duff, P.M.D., Hallam, A. \& Walton, E.K. 1967. Cyclic sedimentation. Elsevier Publishing Company, Amsterdam, 280p.

Estrella, G.O. \& Palagi, P.R, 1969. Plataforma continental de Sergipe-Alagoas: avaliação preliminar da área de Vaza Barris (Fm. Muribeca e Grupo Sergipe). Rel. Interno Petrobrás, Rio de JAneiro, in TOFFOLI, L.C, ed. Margem Continental Brasileira - Coletânea de Relatórios de Exploração, p.83-102.

Feijó, F.J. 1980. Estudo dos carbonatos Muribeca e Riachuelo no Alto de Aracaju - Bacia de Sergipe-Alagoas - nordeste do Brasil. Anais do XXXI Congr.Bras.de Geol. (Camboriú, SC), 1: 320-332.

Fischer, A.G. \& Bottjer, D.J. 1991. Orbital forcing and sedimentary sequences. Journal Sedim. Petrol., 61 (7): 1063-1069

Ginsburg, R.N., 1975. Tidal deposits. A casebook of recent examples and fossil counterparts. Springer-Verlag, Berlin, 428p.

Goldhammer, R.K., Dunn, P.a., \& Hardie, L.A. 1987. High frequency glacio-eustatic sealevel oscilations with Milankovitch characteristics recorded in middle Triassic platform carbonates in northeast Italy. Am. Jour: of Science, 287: 853-892.

Goodwin, P.W. \& Anderson, E.j. 1986. Punctuated aggradational cycles: a general hypotesis of episodic stratigraphic accumulation .
Journal of Geology, 93: 515-533.

Hallam, A., 1981. Facies interpretation and the stratigraphic record. W.H. Freeman \& Co. Ltd., 276p.

Hardie, L.A.\& Shinn, E.A.,1986. Carbonate depositional environments. Modern and recent. Part 3: tidal flats. Quarterly Journal of the Colorado School of Mines, 81(1), 1-74.

Koutsoukos, E.A.M., 1989. Mid to late Cretaceous microbiostratigraphy, paleoecology and paleogeography of the Sergipe basin, northeastern Brazil. Ph.D. thesis, Polytechnic South West, Plymouth, England, 680p.

Koutsoukos, E.A.M.,Mello, M.R.,\& Azambuja Filho, N.C. de 1991 Micropalaeotological and geochemical evidence of mid-Cretaceous dysoxic-anoxic palaeoenvironments in the Sergipe Basin, northeastern Brazil. Geol.Soc.Special Publ.,58,p427-447.

Koutsoukos, E.A.M, Azambuja Filho, N.c. De, Spadini, A.r. \& Destro, N., 1993. Upper aptian - lower coniacian carbonate sequences in the Sergipe Basin, northeastern Brazil. In: Simo,T, Scott,R.W. \& Masse,J.P. (eds.). Atlas of Cretaceous Carbonate Platforms. AAPG (Special Publication), Tulsa, Oklahoma, 11p.

Mendes, J.M.C, 1993. Análise Estratigráfica da Seção Neo-Aptianal Eocenom aniana (Fm. Riachuelo) na área de Aracaju e adjacências - Bacia de Sergipe - Alagoas. Dissertação de mestrado, UFRGS, Porto Alegre, RS, 166p.

Ojeda, H.a.o. \& Bisol, D.L. 1971. Integração geológica regional da extensão submarina da bacia sedimentar de Sergipe-Alagoas. Anais do XXV Congr.Bras. de Geol. (São Paulo, 1971), 3: 215-225.

Ojeda, H.A.O. \& Fujita, A.M. 1976. Bacia Sergipe-Alagoas: geologia regional e perspectivas petrolíferas. Anais do XXVIII Congr. Bras. de Geol. (Porto Alegre, 1974), 1: 137-158.

Ponte, F.C. \& Asmus, H.E. 1976. The Brazilian marginal Basins: current state of knowledge, in ALMEIDA, F.F.M. de, ed., Continental Margins of Atlantic Type, Anais da Acad. Bras. de Ciências, 48 (suplemento): 215-248, Rio de Janeiro.

Purdy, E.G., Pusey Iii, W.C. \& Wantland, K.F., 1975. Continental shelf of Belize - Regional shelf attributes, in Wantland, K.f. \& Pusey III, W.C. (Eds.), Belize Shelf - Carbonate Sediments, Clastic Sediments, and Ecology. AAPG Studies in Geology 2, 599p

Seilacher, A. 1991. Events and their signatures - an overview, in Einsele et al.(Eds.), Cycles and Events in Stratigraphy. Springer-Verlag.

Schaller, H. 1970. Revisão estratigráfica da Bacia de Sergipe-Alagoas. Bol. Técn. Petrobrás, 12 (1): 21-86, Rio de Janeiro

Spadini, A.R. 1992. Processos deposicionais e ciclicidade em carbonatos Albianos de plataforma rasa da Bacia de Campos. Dissertação de Mestrado, Universidade Federal do Rio de Janeiro, Rio de 
Janeiro, 1-115.

Tagliari, C.V. 1993. Evolução das seqüências mistas (siliciclásticas e carbonáticas) sob a influência da halocinese durante o Albo-Aptiano da plataforma de Regência -Bacia do Espírito Santo. Dissertação de Mestrado, Universidade Federal do Rio Grande do Sul, RS, $159 \mathrm{p}$.
Teixeira, A.A., 1969. Bacia marinha de Sergipe: evolução tecto-sedimentar. Rel.Interno Petrobrás, Rio de Janeiro, 26p.

Wilson, J.L. 1975. Carbonate facies in geologic history. Spring-Verlag, New York, 1-471. 